\title{
Fatigue Life Analysis of Cantilever Probe on Wafer Test
}

\author{
Te-Ching Hsiao ${ }^{1, a}$, Hao-Yuan Chang $^{2}$ and Shyh-Chour Huang ${ }^{1}$ \\ ${ }_{2}^{1}$ Department of Mechanical Engineering, National Kaohsiung University of Applied Sciences, Kaohsiung City 807, Taiwan (R.O.C.) \\ 2 Consultant, Sun Home Technology Co., Ltd
}

\begin{abstract}
This research utilizes the finite element analysis software (ANSYS) to stimulate the different probe material quality (tungsten, SUS304 stainless steel, SUS316L stainless steel and SKD11 tool steel, respectively) during wafer tests. Under a room temperature of $\left(25^{\circ} \mathrm{C}\right)$, the stress and fatigue life (cycles of probing test) of the cantilever probe were measured with an OverDriver (OD) of $20 \mu \mathrm{m} \cdot 40 \mu \mathrm{m} \cdot 50 \mu \mathrm{m} \cdot 60 \mu \mathrm{m}$ and $80 \mu \mathrm{m}$, respectively. First, to obtain the magnitude of pinpoint shift of the probe under wafer test and the OverDriver is $50 \mu \mathrm{m}$. And, calculate the fatigue life of the probe. Then, a probe model with the same characteristics as the experiment is created and the probe fatigue life analyzed with the ANSYS. After the reliability of the model is ascertained, the wafer tests of different probe materials are stimulated under different OverDriver circumstances to calculate its stress and fatigue life. The results indicate that the greatest stress measured during the wafer test of the tungsten, SUS304 stainless steel, SUS316L stainless steel and SKD11 tool steel cantilever probe are all smaller than the yield strength, and the fatigue life could reach over one hundred $\mathrm{K}$ cycles. When catalogued by the cantilever probe fatigue life during one hundred $\mathrm{K}$ cycles, the life span, in order, is tungsten $<$ SUS316L stainless steel $<$ SUS304 stainless steel $<$ SKD11 tool steel.
\end{abstract}

\section{Introduction}

In recent years, semiconductor technology advances, from 1990s micron process evolve to today's nanometer manufacturing process, an integrated circuit also trends compact size and functional diversification. In order to improve IC yields, reduce costs, before wafer package, each die should be executed with wafer test, through touch between the probe card and grain specific pad, exposure to the amount of the sensing circuit electrical signals, and then filtered out in advance before packing poor electrical function of the wafer, in order to avoid defective products increased manufacturing costs.

When the wafer testing, pad dimension increased and contact with the probe then subjected to a fixed itinerary, and through a fixed voltage and current to measure its electrical signals. However, when the wafer and probe will cause pad surface scratches, without proper overdrive, may cause unable to measure electrical signals or scratches is too big, too deep, and the underlying cause pad micro-structural damage, arising from the subsequent packaging and reliability problems. In addition, use of the process, the probe was subjected to mush cyclic load, will cause the elastic fatigue, so that the probe tip offset, resulting in pads is overkill.

In 1998, Pandey and Higgins [1] propose a new P4 probe card technology (Photolithograhic Pattern Plated Process), this card is suitable for wafer testing high pin count and high speed, and when overdriving, stroke at $100 \mu \mathrm{m}$, produced aluminum pads scratches size between $10 \mu \mathrm{m} \sim 25 \mu \mathrm{m}$. In 2000 Maekawa [2] et. used tungsten

\footnotetext{
${ }^{\mathrm{a}}$ Corresponding author: author@e-mail.org
}

probe as the test specimen, to compare the influence of different tip shapes (Radius Tip Probe and Flat Tip Probe) contact stability of resistance. The results show radius tip probe more stable by flat tip probe.

In 2001, Hotchkiss [3] and others to measure the number of different probe $(1 \times, 2 \times, 3 \times, 4 \times)$, explore the influence of reliability the scratches on the drawn wire bonding structure fitted. The results show that when the proportion of scratches more than $20 \%$, the combined strength and reliability of configuration of the wire will be significantly reduced. In 2003 Sauter [4], who experimented wafer testing with the thickness of $1.2 \mu \mathrm{m}$ aluminum pad and found that the depth of the scratch of pad should not exceed $0.6 \mu \mathrm{m}$, if for the $45 \mu \mathrm{m}$ diameter solder balls, solder ball indentation size can't exceed $25 \%$ of the solder ball bottom area $25 \sim 30 \%$, otherwise it will affect the combined strength of wire bonding and reduce the reliability of the packaging process.

In 2005, Liu and Desbiens [5] etc. , who investigate the structure for aluminum pad, copper pad and tungsten probe, Tungsten-Rhenium probe and overdriving stroke analysis. Besides, use nonlinear finite element to simulate the probe tip contact with the pad surface, the article mentioned that the biggest scratch length calculation, the safe distance should remain must be $5 \sim 8 \mu \mathrm{m}$. Further, the probe tip and the pad contact surface is smaller, the deformation of pads can be reduced.

In 1954, Coffin [6] and Manson [7] set up plastic strain-life theory, which was investigated the fatigue damage problem caused by thermal stress and the high 
stress load, advanced the empirical relationships between load times and the plastic strain in fatigue damage, according to the strain, to describe the fatigue was widely used, which is called Coffin-Manson relationship.

In 1998, Yasushi Miyano [8] et al proposed the following four basic assumptions used to predict the fatigue life, (1) at stationary strain rate, creep rate and fatigue damage, its destroyed structure is the same. (2) in the same time - the temperature, all breaking strength have the principle of superposition. (3) for a single load, it may use the laws of linear cumulative damage. (4) $\mathrm{s}$ tress ratio of linear fatigue have dependencies. With the above four assumptions, at a stationary strain rate, creep rate, frequency and stress ratio, with translational and rotational shift factor in the SN curve, to find an optimal master curve.

In this overdriving stroke is $50 \mu \mathrm{m}$, the size of the scratch of aluminum pad, the fatigue life of the tungsten cantilever probe design will be calculated and calculate the maximum stress and fatigue life of the probe by finite element analysis software. After the simulation result and the experimental values are correspond, execute the analysis of maximum stress and fatigue life with different overdriving stroke (respectively $20 \mu \mathrm{m}, 40 \mu \mathrm{m}, 50 \mu \mathrm{m}$, $60 \mu \mathrm{m}$ and $80 \mu \mathrm{m}$ ), different probe material (respectively tungsten, low-carbon steel, SUS304 stainless steel, SUS316L stainless steel and SKD11 tool steel). The results can be used as a reference in probe design and through appropriate probe design to improve the life of the probe.

\section{Theory of fatigue life}

When structure repeated loads, will form a cyclic strain and stress, even if stress is less than the yield strength of the material, it will lead to the destruction of the structure, this is the fatigue damage. In the course of the fatigue study, in earlier studies focused on high cycle fatigue $(\mathrm{N}$ $>10^{4} \sim 10^{5}$ ) discussion, the corresponding fatigue curve that is the stress amplitude and cycle curve (S-N curve) to describe the fatigue properties of material. But as the study of low-cycle fatigue increased attention and needs to find in such a short life to explore the issue, $\mathrm{S}-\mathrm{N}$ curve can't meet the requirements, and strain-controlled fatigue theory closer to the actual situation, and to avoid the stress under control unstable or out of control situations.

\subsection{The relationship of Plastic stress - strain}

Material by uniaxial cyclic loading, the plastic stress strain relationship [14]:

$$
\sigma=K^{\prime}\left(\varepsilon_{p}\right)^{n^{\prime}}
$$

In this case, $\sigma$ is the cyclically stress amplitude, $\varepsilon_{\mathrm{p}}$ is cyclically plastic strain amplitude, $\mathrm{K}^{\prime}$ is cyclically strength coefficient, $\mathrm{n}^{\prime}$ is the cyclically strain hardening exponent, generally $\mathrm{n}^{\prime}$ is referred between 0.1 and $0.25[14]$. The total strain is elastic strain and plastic strain sum and the equation of total strain can be obtained by the Hook's law.

$$
\varepsilon=\varepsilon_{e}+\varepsilon_{p}=\frac{\sigma}{E}+\left(\frac{\sigma}{K^{\prime}}\right)^{\frac{1}{n^{\prime}}}
$$

Loading cycle repeated, $\Delta \sigma$ and $\Delta \varepsilon$ is the total amount of change of the stress and strain, so that (2) is rewritten as

$$
\frac{\Delta \varepsilon}{2}=\frac{\Delta \sigma}{2 E}+\left(\frac{\Delta \sigma}{2 K^{\prime}}\right)^{\frac{1}{n^{\prime}}}
$$

Figure 1 is a material subjected to cyclic stress, the relationship between stress and strain of elastoplastic.

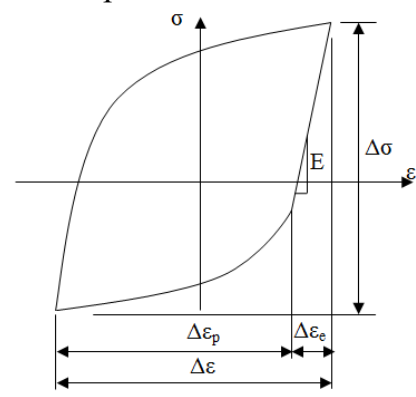

Figure 1. In cyclic stress, the relationship between stress and strain of elastoplastic.

\subsection{Strain-Life Curve}

In fatigue analysis, the strain-life approach is used of the material nature of fatigue and strain - life curve to evaluate material life; and in the elastic range, the fatigue life can be expressed as [14] :

$$
\frac{\Delta \varepsilon_{e}}{2}=\frac{\Delta \sigma}{2 E}=\frac{\sigma_{f}^{\prime}}{E}\left(2 N_{f}\right)^{b}
$$

In this equation : $\Delta \varepsilon_{\mathrm{e}} / 2$ is true strain amplitude, $\Delta \sigma / 2$ is true stress amplitude, $\sigma_{\mathrm{f}}^{\prime}$ is fatigue strength coefficient, $2 \mathrm{~N}_{\mathrm{f}}$ is reversals to failure ( $1 \mathrm{rev}=0.5$ cycle $), \mathrm{b}$ is fatigue strength exponent, in general, $\mathrm{b}$ is between -0.05 to -0.14 $[9,14]$.In the plastic strain range, the fatigue life can be expressed as [14] :

$$
\frac{\Delta \varepsilon_{p}}{2}=\varepsilon_{f}^{\prime}\left(2 N_{f}\right)^{c}
$$

In this equation: $\Delta \varepsilon_{\mathrm{p}} / 2$ is plastic strain amplitude, $\varepsilon_{\mathrm{f}}^{\prime}$ is fatigue ductility coefficient, $\mathrm{c}$ is fatigue ductility exponent, in general, $\mathrm{c}$ is between -0.5 to $-0.7[9,14]$.

The total strain amplitude is the sum of elastic strain and plastic strain, it can be written as

$$
\frac{\Delta \varepsilon}{2}=\frac{\sigma_{f}^{\prime}}{E}\left(2 N_{f}\right)^{b}+\varepsilon_{f}^{\prime}\left(2 N_{f}\right)^{c}
$$

Coffin-Manson formula is estimating formula, equation (6) of the strain - fatigue life curve shown in Figure 2. 


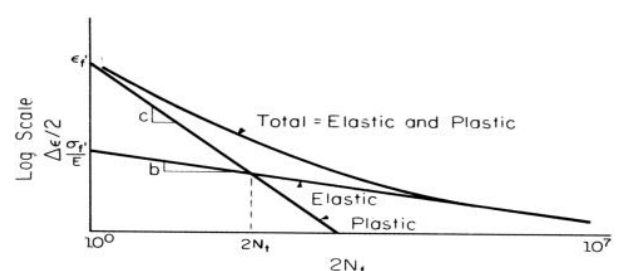

Figure 2. The strain - fatigue life curve

In recent years, experimental evidence accumulated by a number of researchers showed the correlation between cycle life and total strain amplitude ratio is better than the one between the plastic strain amplitude, but the fatigue life relationships in actual project need to go through another amendment. In this paper, use Morrow correction theory, the simulate fatigue life can approach real fatigue life, the corrected strain fatigue life is showed following equation :

$$
\frac{\Delta \varepsilon}{2}=\frac{\sigma_{f}^{\prime}-\sigma_{m}}{E}\left(2 N_{f}\right)^{b}+\varepsilon_{f}^{\prime}\left(2 N_{f}\right)^{c}
$$

In this equation: $\sigma_{\mathrm{m}}$ is average stress amplitude.

\section{The parameters of test specimen}

In wafer test, cantilevered probe direct contact with aluminum pads, and aluminum pads will be left permanent scratches, but also because of repeated use of the probe, and fatigue damage.

\subsection{Probe geometry}

In this paper, the geometry and dimensions of the cantilever probe are respectively shown in Figure 3 and Table 1. The more important factors which affect the structural strength of the probe are beam length, bending angle, shooting angle and probe diameter and so on.

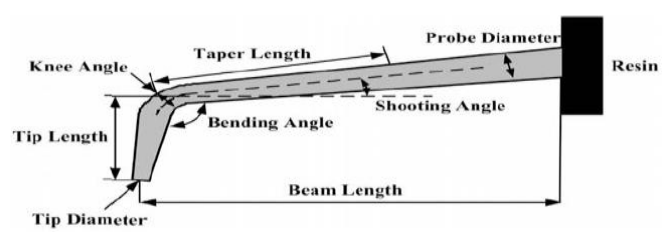

Figure 3. Cantilever probe schematic

Table 1. Cantilever probe geometry

\begin{tabular}{|c|c|}
\hline Probe Diameter $(\mu \mathrm{m})$ & 150 \\
\hline Beam Length $(\mu \mathrm{m})$ & 2,500 \\
\hline Knee Diameter $(\mu \mathrm{m})$ & 45 \\
\hline Tip Diameter $(\mu \mathrm{m})$ & 23 \\
\hline Tip Length $(\mu \mathrm{m})$ & 279.4 \\
\hline Bending Angle $\left(^{\circ}\right)$ & 103 \\
\hline Shooting Angle $\left.^{\circ}\right)$ & 8 \\
\hline${\text { Taper Length }\left(\mathrm{um}^{\circ}\right)}$ & 1,750 \\
\hline
\end{tabular}

\section{2 pad geometry}

The geometric shapes and sizes of aluminum pad and the silicon substrate were used as shown in Figure 4. When the wafer testing, fixed probe handle (at maximum diameter), then rises up the loading silicon substrate. After the pad in contact with the probe, and then rises to the set of overdrive, and silicon substrate returned to the original position. After an overdrive was completed, probing actions will leave permanent scratches in the welding pad.

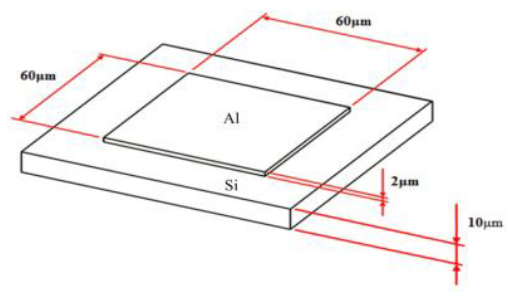

Figure 4. Geometry and dimensions of the pad

\subsection{Related properties parameters of material}

Nowdays, the most commonly probe material was used for wafer testing is tungsten, this article the starting point is angle of fatigue life, attempting to analyze other possible materials of metal of probe, where the choice of 304 stainless steel, 316L stainless steel and SKD11 tool steel, etc. The mechanical properties of each material shown in Table 2 [10], the analysis of fatigue about the material parameters required for as shown in Table 3 [10$13]$.

Table 2. The mechanical properties of material [10]

\begin{tabular}{|c|c|c|c|c|}
\hline Materials & Tungsten & SUS304 & SUS316L & SKD11 \\
\hline Density $\left(\mathrm{kg} / \mathrm{m}^{3}\right)$ & 19,300 & 8,000 & 7,800 & 8,400 \\
\hline $\begin{array}{c}\text { ElasticModulus } \\
(\mathrm{GPa})\end{array}$ & 290 & 193 & 193 & 208 \\
\hline Poisson's Ratio & 0.28 & 0.29 & 0.29 & 0.30 \\
\hline $\begin{array}{c}\text { Yield Strength } \\
(\mathrm{MPa})\end{array}$ & 1770 & 405 & 365 & 585 \\
\hline $\begin{array}{c}\text { Ultimate Strength } \\
(\mathrm{MPa})\end{array}$ & 2600 & 505 & 822 & 668 \\
\hline
\end{tabular}

Table 3. The fatigue parameters of material [10-13]

\begin{tabular}{|l|c|c|c|c|}
\hline \multicolumn{1}{|c|}{ Material } & Tungsten & SUS304 & SUS316L & SKD11 \\
\hline $\begin{array}{l}\text { Fatigue Strength } \\
\text { Coefficient(MPa) }\end{array}$ & 1,020 & 1,100 & 997 & 890 \\
\hline $\begin{array}{l}\text { Fatigue Strength } \\
\text { Exponent }\end{array}$ & -0.09 & -0.12 & -0.11 & -0.09 \\
\hline $\begin{array}{l}\text { Fatigue Ductility } \\
\text { Coefficient }\end{array}$ & 0.5 & 0.34 & 0.47 & 0.51 \\
\hline $\begin{array}{l}\text { Fatigue Ductility } \\
\text { Exponent }\end{array}$ & -0.54 & -0.49 & -0.51 & -0.59 \\
\hline $\begin{array}{l}\text { Cyclic Strength } \\
\text { Coefficient (MPa) }\end{array}$ & 1,300 & 1,424 & 1,232 & 986 \\
\hline $\begin{array}{l}\text { Cyclic Strain } \\
\text { Harding Exponent }\end{array}$ & 0.16 & 0.24 & 0.23 & 0.15 \\
\hline
\end{tabular}




\section{The probe tip offset analysis}

When the wafer testing, the offset tip result from the probe material fatigue, Figure 5 is a schematic view of a probe offsets. Experiments that use tungsten cantilever probe in overdrive stroke under $50 \mu \mathrm{m}$, the relationship between tip offset and cycles times as shown in Table 4; and Figure 6 is a relationship diagram about tip offset and cycles times, by the relationship between tip offset and cycles times, use the least squares method, we can calculate the equation related between the tip offset and Probing shown as.

$$
Y=0.0167 X+0.068
$$

In this equation, $\mathrm{Y}$ is the offset of $\operatorname{tip}(\mu \mathrm{m}), \mathrm{X}$ is probing times $\left(10^{3}\right)$

According to the relevant literature, in order to maintain the reliability when the chip package, when the wafer testing scratches length must be less than $20 \%$ of the length of the pad [3], and scratches must distance of 5 $\sim 8 \mu \mathrm{m}$ border security. In response to the practice of tip offsets of probe, the tip of the probing the initial placement position control in one-third of the length, so calculate the tip offset arising due to fatigue and allow a maximum of $10 \mu \mathrm{m}$. Then according to (5) calculation, the fatigue life of probe design is $5.95 \times 10^{5}$ times. Figure 7 is a tungsten probe and aluminum pad in a stroke of $50 \mu \mathrm{m}$, the photo of the pad scratches.

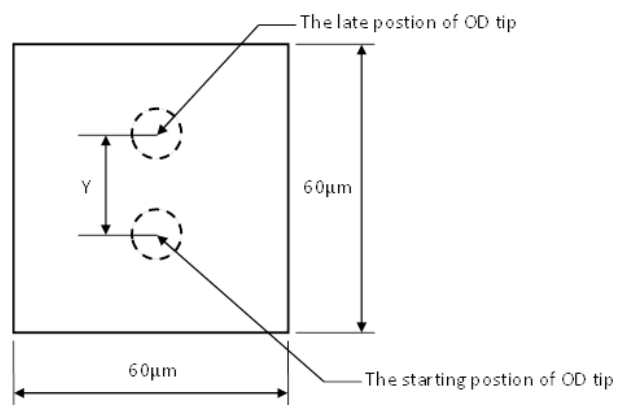

Figure 5. A schematic view of a probe offsets.

Table 4. The relationship between tip offset in Experimention and cycles times.

\begin{tabular}{|c|c|}
\hline Cycles times & Tip offset $(\boldsymbol{\mu m})$ \\
\hline $50 \times 10^{3}$ & 1.12 \\
\hline $100 \times 10^{3}$ & 1.63 \\
\hline $150 \times 10^{3}$ & 2.42 \\
\hline $200 \times 10^{3}$ & 3.52 \\
\hline
\end{tabular}

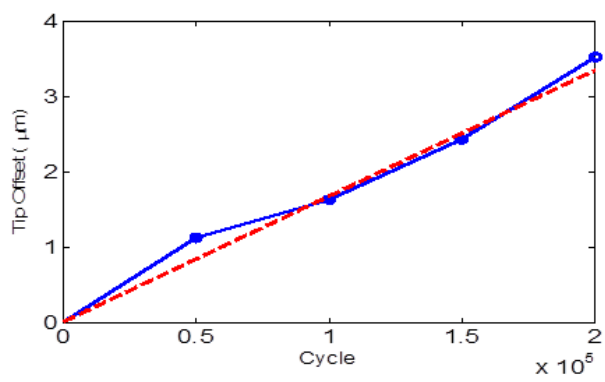

Figure 6. When $\mathrm{OD}=50 \mu \mathrm{m}$, tip offset - cycles times curve

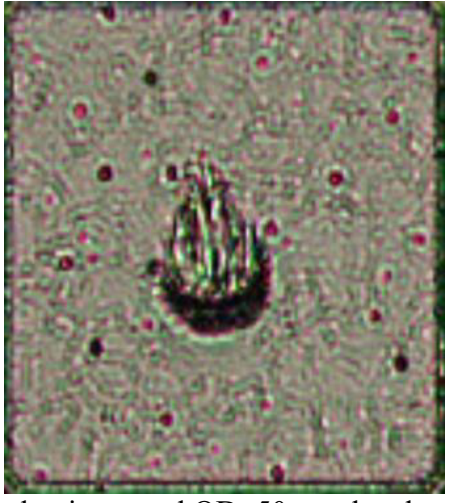

Figure 7. When aluminum pad $\mathrm{OD}=50 \mu \mathrm{m}$, the photo of the pad scratches

\section{The finite element model construction}

\subsection{CAD model construction}

Wafer testing process is supported by the silicon substrate under the pad moves up, after the pads contact on the probe tip, to finish up the set of overdrive, then separated down, returned to the starting position, complete a cycle; pad of length, width and height respectively $60 \mu \mathrm{m}, 60 \mu \mathrm{m}$ and $2 \mu \mathrm{m}$, shown in Figure 3.

\subsection{Model grid and boundary conditions}

Import the CAD model which was completed to the ANSYS analysis module, and go mesh, the model is cut to fit the size, the contact surface between the probe and the pad was more refined to $1 \mu \mathrm{m}$ or less, in order to achieve a more accurate analysis. Set the boundary conditions on the right side of fixed end in Fig. 8, the left side of the silicon substrate and aluminum pads move upward displacement overdrive required. The completion of meshing and boundary conditions set are shown in Figure 8.

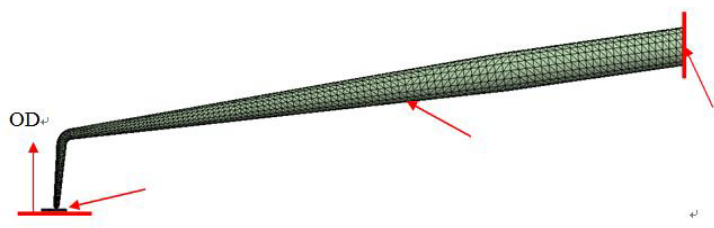

Figure 8. The meshing of OD model and boundary conditions

\subsection{Calculation process of fatigue analysis}

After completing the model mesh, analyzed the using mechanical properties of the material in Table 2, input the finite element to the ANSYS analysis module. In calculating OD process, the mechanical behavior of the probe; after obtaining analysis information of probe on the mechanical behavior, in Table 3 , the material parameters of fatigue and strain - fatigue (equation (1) to (7)) to calculate the fatigue life of the probe throughout the body, and retrieve the minimum value of the fatigue life as the probe design value of fatigue life. Figure 11 is 
the stress amplitude - life (S-N) curve of a tungsten probe that calculated with the information in Table 3.

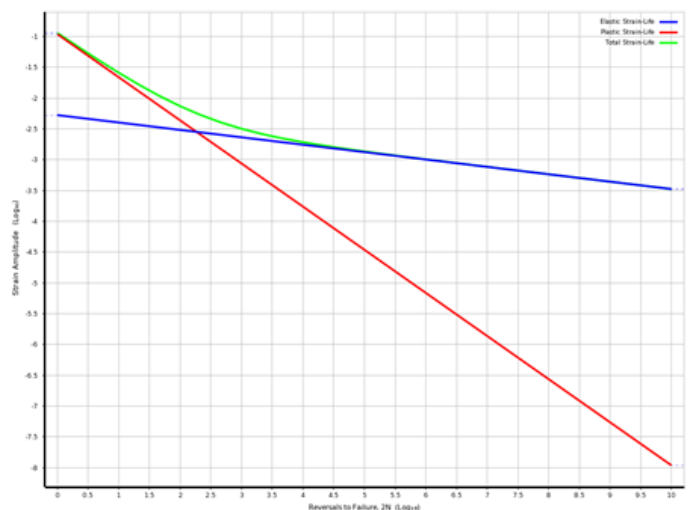

Figure 9. The stress amplitude - life (S-N) curve of a tungsten probe

\section{The analysis result of finite element model}

After model construction completed, run the simulation analysis of finite element in OD stroke is $50 \mu \mathrm{m}$ and calculate the value of fatigue life, and take the results to compare the experimental data, in determining the simulation results and experimental values are similar before further analysis.

\subsection{Stress analysis of OD}

After the ANSYS mesh model was establishd, we can performed OD analysis in OD stroke of $50 \mu \mathrm{m}$, then we found that the maximum stress of the probe is $574.88 \mathrm{MPa}$, the results shown in Figure 10, compare the material properties of tungsten in Table 2 and know the maximum stress of the probe is less than the yield stress of tungsten, it was found that the tip of the offset was resulted from the material fatigue, the obtained analysis from the scratch that on aluminum pad shown in Figure 11.

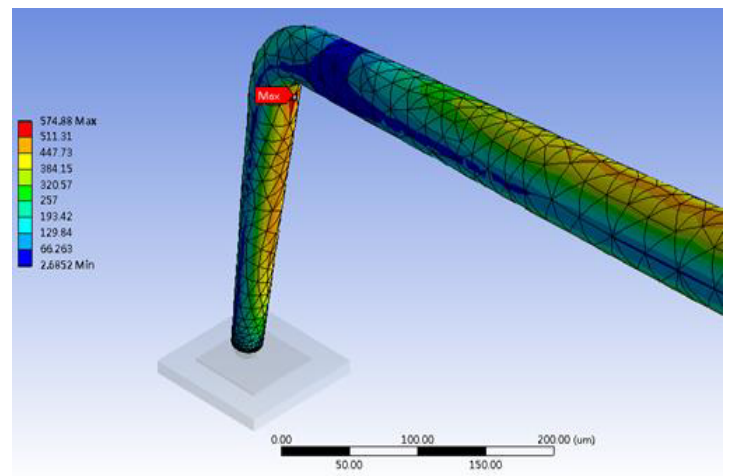

Figure 10. The diagram of the maximum stress of the tungsten probe in $\mathrm{OD}=50 \mu \mathrm{m}$.

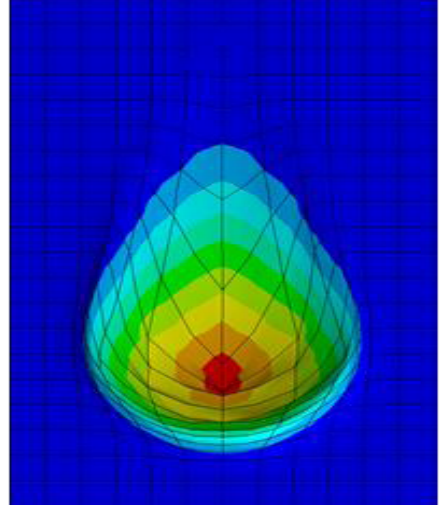

Figure 11. The diagram of the scratch that on aluminum pad in $\mathrm{OD}=50 \mu \mathrm{m}$

\subsection{Fatigue analysis comparison}

Processed the ANSYS analysis of fatigue with the material factor of tungsten metal in Table, and obtain the cycle life is $6.12 \times 10^{5}$ times, is similar to the experimental value is $5.95 \times 10^{5}$ times, which shows the reliability analysis model has good.

\subsection{Simulation Results}

After determining the analytical model, the idea that is based on expanse the type of materials to use, we try to analyze a material, that nobodys use on OD in past, for example SUS304 stainless steel, SUS316L stainless steel and SKD11 tool steel, in the same size for probe and pad and aluminum pad, processed the mechanical behavior of the probe and fatigue analysis.

Table 5 is the variety of materials of probe, in the maximum stress measured at each stroke of OD, the mechanical properties of the material in Table 5 and Table 2 is evident, in the OD stroke less than $60 \mu \mathrm{m}$, in addition to the $316 \mathrm{~L}$ stainless steel, other materials of probe that maximum stress is less than the yield stress. Table 6 and Figure 12 is the variety of materials of probe, according to the maximum stress in Table 5, calculated fatigue life of each stroke of OD.

Table5. The maximum stress of each stroke of OD with a variety of materials of probe (unit:MPa)

\begin{tabular}{|c|c|c|c|c|}
\hline OD Material & Tungsten & SUS304 & SUS316L & SKD11 \\
\hline $20 \mu \mathrm{m}$ & 219.95 & 120.32 & 135.88 & 147.21 \\
\hline $40 \mu \mathrm{m}$ & 435.80 & 243.52 & 261.60 & 284.64 \\
\hline $50 \mu \mathrm{m}$ & 574.88 & 332.72 & 332.40 & 358.02 \\
\hline $60 \mu \mathrm{m}$ & 705.12 & 401.25 & 410.70 & 442.50 \\
\hline $80 \mu \mathrm{m}$ & 915.25 & 502.78 & 537.62 & 577.42 \\
\hline
\end{tabular}

Table 6. The analysis result of fatigue of a variety of materials of probe (cycles)

\begin{tabular}{|c|c|c|c|c|}
\hline $\mathrm{OD}(\mu \mathrm{m})$ & $\mathrm{W}$ & SUS304 & SUS316L & SKD11 \\
\hline 20 & $7.04 \times 10^{9}$ & $7.68 \times 10^{9}$ & $7.01 \times 10^{9}$ & $3.29 \times 10^{10}$ \\
\hline 40 & $3.68 \times 10^{6}$ & $1.88 \times 10^{7}$ & $1.81 \times 10^{7}$ & $5.47 \times 10^{7}$ \\
\hline 50 & $6.12 \times 10^{5}$ & $4.05 \times 10^{6}$ & $3.75 \times 10^{6}$ & $6.08 \times 10^{6}$ \\
\hline 60 & $2.12 \times 10^{5}$ & $1.17 \times 10^{6}$ & $1.27 \times 10^{6}$ & $1.54 \times 10^{6}$ \\
\hline 80 & $7.49 \times 10^{4}$ & $2.41 \times 10^{5}$ & $2.50 \times 10^{5}$ & $6.23 \times 10^{5}$ \\
\hline
\end{tabular}




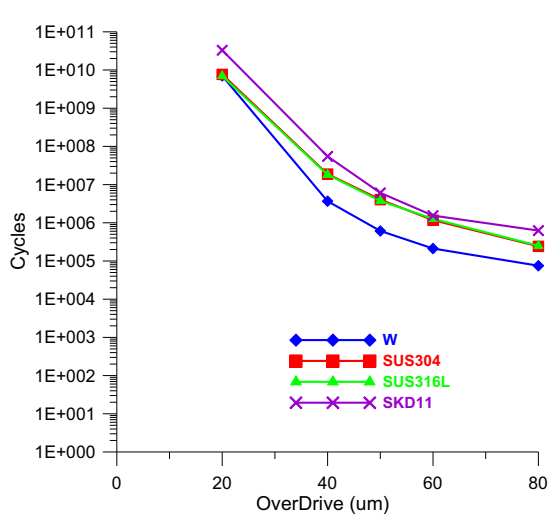

Figure 12. The analysis result of fatigue of a variety of materials of probe

\section{Conclusion}

In this study, we focus on the wafer testing process, analyze the stress is caused in different materials of probe and different stroke of OD and the fatigue life of the probe that under loading cycles. Results from the analysis are summarized several conclusions:

1. The overdriving experiments use tungsten probe that the allowed offset of tip is $10 \mu \mathrm{m}$, the results show that when the OD stroke is $50 \mu \mathrm{m}$, the number of OD will up to $595 \times 10^{3}$ times. In the simulation in the same set situation, the result will become $612 \times 10^{3}$ times, the results differ between expeniment and analysis is only $2.9 \%$.

2. Analysis in different materials showed that the OD stroke is $80 \mu \mathrm{m}$, the maximum stress of material the probe that are tungsten and SKD11 tool steel are less than yield stress, these two kinds of material of the probe did not produce plastic deformation, but when the material are SUS304 and SUS316L stainless steel, if the maximum stress will exceed yield stress, a part of the probe will produce plastic deformation.

3. As shown in Table 6 and Figure 11, the fatigue life of probe for each material from small to large are: Tungsten <SUS316L stainless steel <SUS304 stainless steel $<$ SKD11 tool steel; wherein, SUS316L and SUS304 stainless steel fatigue life considerably. If you consider the fatigue life of the probe, you should use probes SKD11 tool steel for probes that can get the best effect.

\section{References}

1. R. Pandey, D. Higgins, IEEE International Test. Conference, 836-842, (1998)

2. S. Maekawa, M. Takemoto and Y. Kashiba, IEEE ECTC, 1152 - 1156, (2000)

3. G. Hotchkiss, G. Ryan, W. Subido, J. Borz, S. Mitchell, R. Rincon, R. Rolda and L. Guimbaolibot, IEEE ECTC, 1175-1180, (2001)

4. W. Sauter, T. Aoki, T. Hasida, H. Miyai, K.Petrarca, F. Beaulieu, S. Allard, J. Power, and M.Agbesi, IEEE ECTC,(2003)

5. Y. Liu, D. Desbiens, S. Irving, T. Luk, S. Edborg, D. Hahn and S. Park, IEEE ECTC Proceedings, Vol. 1, 861-866, (2005)
6. L.F. Coffin, Transactions of ASME,76,931-950, (1954)

7. S.S. Manson, National Advisory Commission on Aeronautics, Report No. 1170, Cleveland: (1954)

8. Y. Miyano, M. Nakada, H. Kudoh, R. Muki, CFRP. J.Compos.Mater. 34(7), 538-550,(1999)

9. W.W. Lee, L.T. Nguyen, G.S. Selvaduray, Microelectronics Reliability 40, 231-244, (2000)

10. Online Materials Information Resource Mat-Web in http://www.matweb.com/index.aspx

11. A. Bäumel. Jr. and T. Seeger, Elsever Science Publishers. (1990)

12. C. Boller, and T. Seeger, 1(5), Elsevier Science Publishers, Amsterdam.(1987)

13. ANSYS WORKBENCH database.

14. Julie A Bannantine, Jess J Comer, James L Handrock, B. Englewood Cliffs, N.J. Prentice Hall (1990) 